\title{
Shutter Island (2010), el trauma como desencadenante de la esquizofrenia
}

\author{
Josu SEGURA-ABRIL, Iñigo ROLO-BARINAGA, Oscar PARRADO-LÓPEZ \\ UPV/EHU. Leioa, Vizcaya (España). \\ Autor para correspondencia: Oscar Parrado López. Correo electrónico: Oscarparrado10@gmail.com \\ Recibido el 17 de mayo de 2018; aceptado el 18 de junio de 2018.
}

Cómo citar este artículo: Segura Abril J, Rolo Barinaga I, Parrado López O. Shutter Island (2010), el trauma como desencadenante de la esquizofrenia. Rev Med Cine [Internet] 2019;15(1): 37-44.

DOI: http://dx.doi.org/10.14201/rmc.20241

\begin{abstract}
Resumen
La esquizofrenia es una de las enfermedades mentales que más incógnitas plantea, no solo a la comunidad científica sino también al resto de la población; que a lo largo de la historia ha observado cómo la industria del cine y los medios de comunicación han retratado de una forma extravagante a las personas que la padecen. Relacionándolas, a su vez, con la violencia y el consumo de drogas.

Sin tener en cuenta otros factores de riesgo que también actúan como desencadenantes de la enfermedad y sobre los que, como profesionales de la salud, podemos actuar.

El objetivo principal de este análisis, es el de mostrar cómo un suceso traumático puede actuar como desencadenante de una enfermedad mental, como la esquizofrenia.

Para ello, se realizó un análisis de la película, Shutter Island, a través del visionado de esta. Además, se emplearon varios fotogramas para hacer énfasis en los aspectos relacionados con el objetivo. Por otra parte, se realizaron diversas búsquedas de información vinculadas con la temática de la esquizofrenia y otras patologías asociadas.
\end{abstract}

Palabras clave: esquizofrenia, trauma, patología, estrés postraumático, enfermedad mental, psiquiatría.

\section{Shutter Island (2010). Trauma as a trigger for schizophrenia}

\author{
Summary \\ Schizophrenia is one of the mental illnesses that more questions arise, not only to the scientific community but also \\ to the rest of the population; that throughout history has observed how the film industry and the media have por- \\ trayed extravagantly to the people who suffer it. Relating them to violence and drug use. \\ Without taking into account other risk factors that also act as triggers of the disease and on which we can act as \\ health professionals. \\ The main objective of this analysis is to show that mental illnesses, such as Schizophrenia, can be triggered by trau- \\ matic event. \\ For this, an analysis of the film, Shutter Island, was made through viewing it. In addition, several frames were used \\ to emphasize the aspects related to the objective. On the other hand, several searches for information linked to the \\ topic of schizophrenia and other associated pathologies have been found.
}

Keywords: Schizophrenia, Trauma, Pathology, Posttraumatic stress, Mental illness, Psychiatry.

Los autores declaran que el artículo es original y que no ha sido publicado previamente. 
Ficha técnica

Título: Shutter Island.

Título original: Shutter Island.

Otros título: en países hispanohablantes $L a$ isla siniestra.

País: Estados Unidos.

Año: 2010.

Director: Martin Scorsese.

Música: varios.

Fotografía: Robert Richardson.

Montaje: Thelma Schoonmaker.

Guión: Laeta Kalogridis (Novela: Dennis Lehane).

Intérpretes: Leonardo DiCaprio, Mark Ruffalo, Ben Kingsley, Emily Mortimer, Michelle Williams, Patricia Clarkson, Max von Sydow, Jackie Earle Haley, Elias Koteas, John Carroll Lynch, Ted Levine, Christopher Denham, Robin Bartlett, Nellie Sciutto, Ruby Jerins, Jill Larson.

Color: color.

Duración: 138 minutos.

Género: thriller, intriga, thriller psicológico, años 50.

Productoras: Paramount Pictures, Phoenix Pictures, Sikelia Productions, Appian Way.

Sinopsis: "En el verano de 1954, los agentes judiciales Teddy Daniels (DiCaprio) y Chuck Aule (Ruffalo) son destinados a una remota isla del puerto de Boston para investigar la desaparición de una peligrosa asesina (Mortimer) que estaba recluida en el hospital psiquiátrico Ashecliffe, un centro penitenciario para criminales perturbados dirigido por el siniestro doctor John Cawley (Kingsley). Pronto descubrirán que el centro guarda muchos secretos y que la isla esconde algo más peligroso que los pacientes. Thriller psicológico basado en la novela homónima de Dennis Lehane (autor de "Mystic River" y "Gone Baby Gone")." (Tomado de www.filmaffinity.com).

Premios: 2010: National Board of Review: Top 10 mejores películas, mejor diseño 2010: Festival de Berlín: Sección oficial de largometrajes a concurso. 2010: Satellite Awards: Nominada Fotografía, Sonido, Montaje y Dirección Artística. 2010: Asociación de Críticos de Chicago: Nominada Mejor Fotografía . 2010: Saturn Awards: 5 nominaciones incluyendo Película Terror / Thriller. 2010: Sociedad de Editores de Sonido: Nominada a

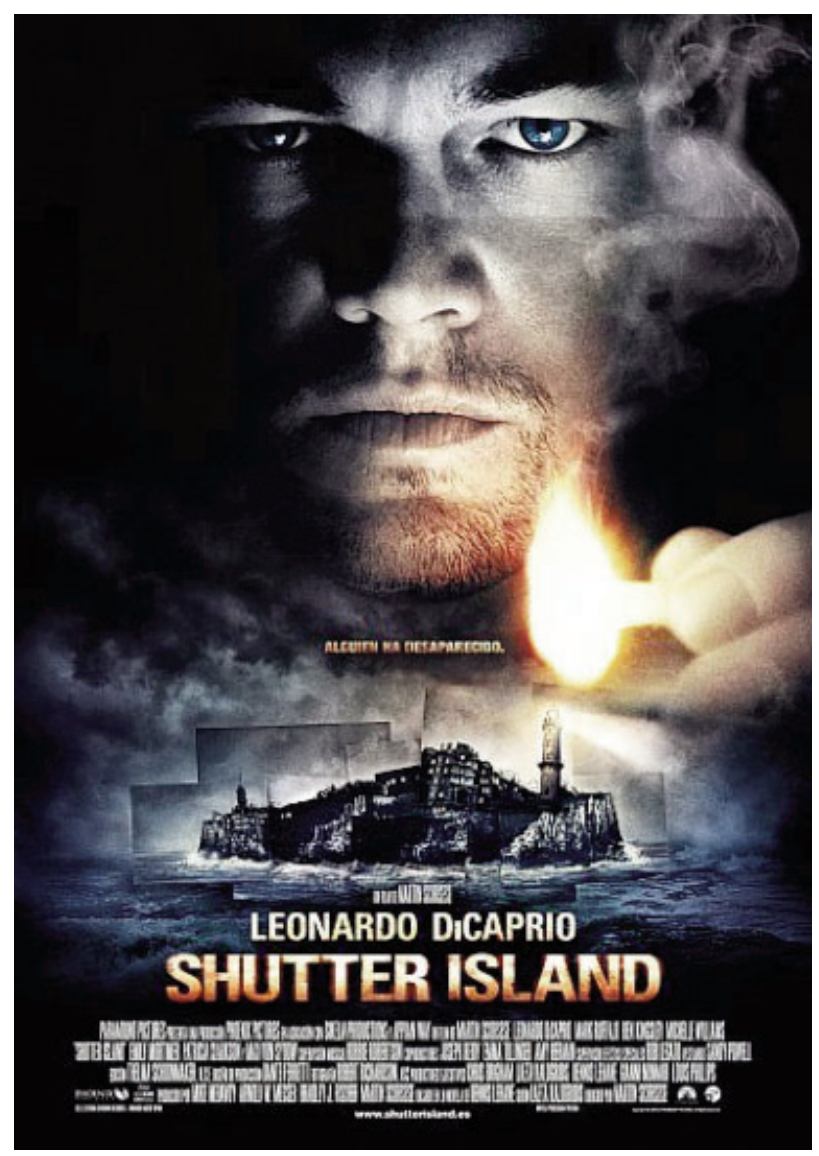

Mejor Sonido. 2010: Sociedad de Críticos de Las Vegas: Nominada Mejor Montaje. 2010: Sociedad de Críticos Online: Nominada Mejor Fotografía. 2010: Sociedad de Críticos de Phoenix: Nominada Mejor Película y Montaje. 2010: Sociedad de Críticos de San Diego: Mejor Diseño de Producción. 3 nom. 2010: Sindicato Italiano de Periodistas de Cine: Nominada Mejor Director no europeo.

Enlaces:

http://www.shutterisland.com/

http://www.imdb.com/title/tt1130884/?ref =ttfc fc tt https://www.filmaffinity.com/es/film173696.html\#

\section{Tráiler en español}

Introducción

La esquizofrenia es, actualmente, una de las enfermedades mentales más conocidas por la sociedad. A pesar de ello, la mayoría de las personas serían incapaces de describir los síntomas o características más comunes ${ }^{1}$. Además, esta enfermedad aún presenta ciertas 
incógnitas, incluso para la comunidad científica, como por ejemplo la etiología exacta de la enfermedad².

Se trata de una enfermedad psicótica grave y deteriorante que se caracteriza principalmente por alteraciones en el pensamiento, las percepciones, la afectividad, el funcionamiento social, el personal y el laboral. Así mismo, es una enfermedad crónica, por lo que necesita un seguimiento de por vida y unos cuidados basados en el tratamiento farmacológico y psicosocial ${ }^{3}$.

Los pacientes que la padecen sufren periodos de remisión y fases agudas denominadas brotes. Estos se caracterizan por una distorsión del pensamiento, las percepciones, las emociones, el lenguaje, la conciencia de sí mismo y la conducta. Algunas de las experiencias más comunes y llamativas son los síntomas positivos; especialmente: alucinaciones, delirios y la conducta extravagante ${ }^{3}$.

Se calcula que es una enfermedad que afecta a menos del $1 \%$ de la población mundial, es decir, algo más de 21 millones de personas la padecen ${ }^{4}$. En España se concluyó que la frecuencia de esquizofrenia y trastornos afines estaría alrededor de 7 de cada 1.000 habitantes, similar a la hallada en estudios internacionales 5 . Por su parte, en el País Vasco la tasa bruta total de esquizofrenia (1992-2009) fue de 2,99 casos por cada 1.000 habitantes, siendo de 3,08 para Bizkaia, 3,55 para Gipuzkoa y 1,48 para Araba. La incidencia total es de 0,17 casos por cada 1.000 habitantes $^{6}$.

Abordando la problemática desde el punto de vista cinematográfico, lo cierto es que la esquizofrenia es un trastorno abusivamente usado en el cine, la mayoría de veces representado de manera excesivamente "teatral", sin atender a la verdadera sintomatología, dando una falsa imagen al espectador sobre el padecimiento de esta dura enfermedad y convirtiendo al personaje que la padece en un cliché con comportamiento estrambótico y violento.

Este abordaje de la figura de la esquizofrenia a lo largo de la historia del cine, con películas tales como Alguien voló sobre el nido del Cuco / One Flew Over the Cuckoo's Nest (1975) Milos Forman, Una mente maravillosa / A beautiful Mind (2001) Ron Howard, o Cisne negro / Black Swan (2010) Darren Aronofsky, ha fomentado en la sociedad la asociación de esta enfermedad con la agresividad y violencia. Sin embargo, como bien reflejan los últimos datos, menos del 9\% de los ataques violentos han sido cometidos por esquizofrénicos, por lo que su magnitud es pequeña al compararla con el resto de la sociedad7. "De hecho, lo más común es que los pacientes cometan auto-agresiones, ya que el $20-40 \%$ de las personas con esquizofrenia presentan ideación suicida, el $30 \%$ cometen alguna tentativa y el $4-10 \%$ muere por suicidio $8 . "$

El problema radica en que los actos violentos llevados a cabo por estas personas resultan inexplicables para el resto de la población y generan una alarma social importante que contribuye en su estigmatización².

Esta visión negativa o estigmatización de la enfermedad se acentúa con el desconocimiento acerca de esta, concibiendo a las personas que la padecen como violentas, incontrolables e impredecibles; motivando y justificando en muchas ocasiones la violación de sus derechos humanos, tanto en las instituciones de salud mental como en las comunidades ${ }^{9}$. Llegando a los extremos en los que se encuentra nuestra sociedad actual, donde más del $50 \%$ de los esquizofrénicos no reciben una atención adecuada a nivel mundial. En los países más desfavorecidos esta cifra se eleva hasta el $90 \% 4$.

La estigmatización en la esquizofrenia y en la enfermedad mental en general, es uno de los temas que se reflejan en "Shutter Island". Además, al estar ambientada en los años 50, se observan las diferentes posiciones de los psiquiatras y se puede apreciar la diferencia en el tratamiento con respecto a la postura actual.

Lo que llama realmente la atención, y es el motivo de elección de esta película para llevar a cabo el presente análisis, es cómo trata el origen de esta enfermedad; es decir, el hecho que desencadena la enfermedad en la mente del protagonista.

La película muestra el desarrollo de la enfermedad, con sus diferentes síntomas característicos; pero lo que realmente la hace especial y la diferencia de otras de temática similar, es que se presenta en el contexto de un suceso traumático, en el que la mente del protagonista trata de olvidar lo ocurrido. Por tanto, abordando el complejo tema de la esquizofrenia desde el punto de vista de su etiología; que como se ha mencionado anteriormente, sigue planteando incógnitas para numerosos autores, la mayoría de estos dividen su origen principalmente en tres tipos de factores de riesgo: biológicos, genéticos, y psicosociales ${ }^{10}$.

Además, se considera que este último, el psicosocial, es un factor etiológico fundamental, puesto que cuanto más se conozca sobre él, mayor capacidad de abordaje como profesionales se tendrá sobre la enfermedad; incidiendo en la prevención, tratamiento y recuperación ${ }^{10}$. 
Es por esto que, mediante este análisis, se pretende lograr el objetivo de mostrar cómo un suceso traumático puede actuar como desencadenante de una enfermedad mental, como la esquizofrenia.

De igual manera, se quiere reflejar como Scorsese muestra, mediante su talento cinematográfico, la sorprendente capacidad de la mente humana para tratar de aliviar el dolor que supone enfrentarse a la cruel realidad. Todo ello a través de una serie de contradictorias alucinaciones, delirios e ilusiones perfectamente aunadas en un hilo argumental magistral que no solo entretiene al espectador, sino que además muestra desde el punto de vista de la psiquiatría cómo funciona la mente de una persona tras un suceso traumático de tal magnitud.

Por tanto, será la relación entre trauma y esquizofrenia la base fundamental de nuestro análisis y una de las principales razones de elección de esta película. Puesto que se trata de una asociación diferente a lo que la mayoría de la filmografía refleja como consecuencia de un suceso traumático.

\section{Análisis}

El cine, desde sus inicios con el cinematógrafo de los hermanos Lumière en 1895 , tenía como intención la creación de sensaciones en el espectador, al igual que ocurría con el resto de artes. En un comienzo, y debido a lo rudimentario de la recién nacida técnica, estas películas retrataban situaciones comunes de la época como sucede en La salida de la fábrica / La Sortie de l'usine Lumière à Lyon (1895) Louis Lumière o La llegada del tren / L'Arrivée d'un train en gare de La Ciotat (1895) Louis Lumière. Pero rápidamente, los artistas del séptimo arte comenzaron a interesarse por la recreación filmada de sueños y motivos más relacionados con la mente.

Desde entonces, el cine se ha popularizado globalmente y hemos podido asistir a una de las evoluciones artísticas más activas jamás ocurrida en relación con el resto de artes.

En 1942 nacerá en Nueva York uno de los iconos de la historia del cine, uno de los pocos directores que han conseguido entrar en el hipotético "salón de la fama" del séptimo arte estando aún con vida. Nos referimos a Martin Scorsese, sin duda uno de los grandes influyentes en la industria cinematográfica actual.

Conocido especialmente por retratar lo más sórdido del comportamiento humano, tomando por escenario las sucias calles de su idealizada ciudad de Nueva
York, lo cierto es que encontramos verdaderas joyas en su filmografía. Muchas de estas son un acercamiento riguroso a la psique de los personajes, por lo que los trastornos psicológicos son un gran recurrente en sus películas. Desde la depresión en Taxi driver / Taxi driver (1976) Martin Scorsese, hasta la adicción en El lobo de Wall Street / The wolf of Wall Street (2013) Martin Scorsese, pasando por el trastorno obsesivo-compulsivo en El aviador / The Aviator (2004) Martin Scorsese, y por la esquizofrenia en Shutter Island.

Es esta última la película analizada, no solo porque aborda el tema de la esquizofrenia, sino también porque deja margen al espectador para poder interpretar la causa, transcurso y resolución de la problemática planteada.

Para poder comenzar con el análisis es necesario llevar a cabo una descripción general del protagonista y de la historia.

Edward "Teddy" Daniels es un detective que acude al hospital psiquiátrico Ashecliffe, situado en una isla llamada Shutter Island a la que solo se puede acceder en barco. Junto a él, le acompañará su nuevo compañero, Chuck Aule. Su objetivo será el de investigar la desaparición de Rachel Solando, la cual ha desaparecido en extrañas circunstancias.

Durante su estancia allí, comienza a sufrir flashbacks sobre la segunda guerra mundial, en la que había participado en uno de los escuadrones encargados de liberar un campo de concentración en Alemania; y sueños repetitivos en los que aparece su mujer, Dolores Chanal, la cual había muerto en un incendio provocado por Andrew Laedis, el que fuera el bedel de su edificio y personaje al que se describe como pirómano y con un aspecto físico caracterizado por una gran cicatriz que le cruza el rostro y con ojos de diferente color. Es en una de estas ensoñaciones cuando Teddy empieza a creer que Andrew se encuentra en la isla.

A medida que avanza la película descubrimos que Teddy Daniels es en realidad un anagrama de Andrew Laedis, y que esa disociación de personalidad se debe a un intento desesperado de su mente por tratar de ocultar y evitar los terribles recuerdos que a lo largo del metraje se le van mostrando a modo de imágenes distorsionadas, delirios y alucinaciones (Fotos 1, 2, 3 y 4).

En referencia a los episodios citados, se puede comprobar que Teddy experimenta una combinación de síntomas pertenecientes a diferentes patologías 

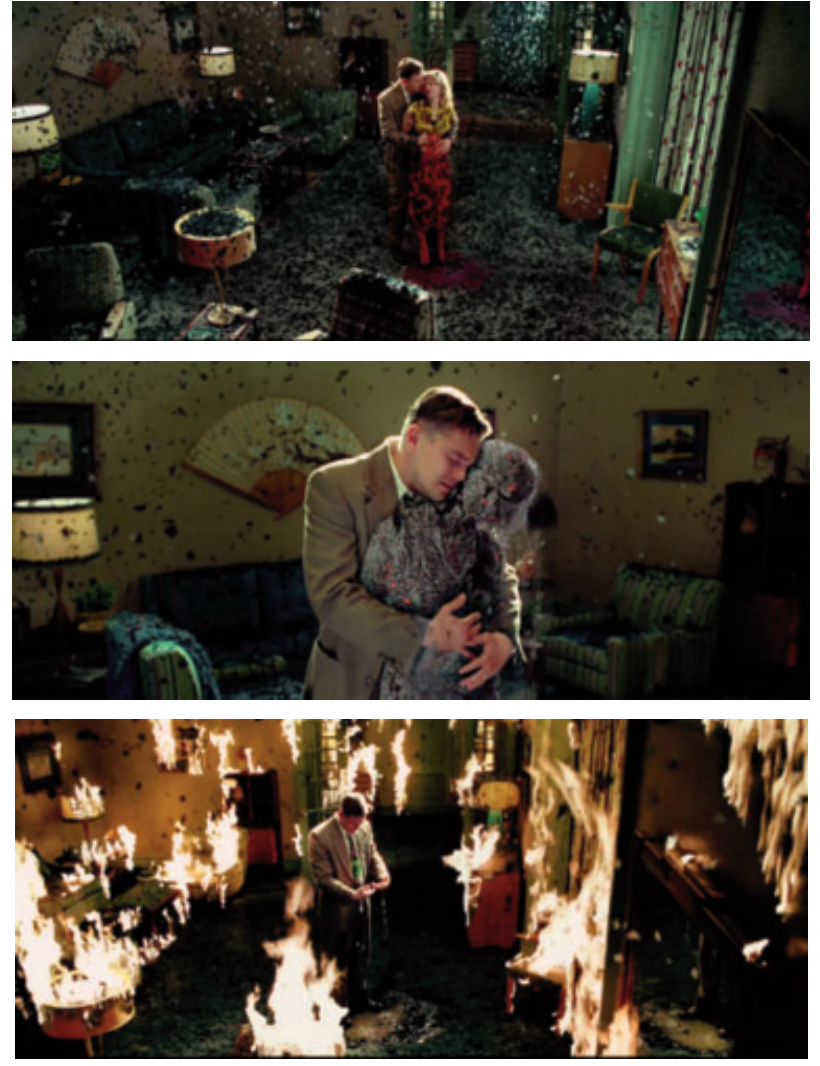

Fotos 1,2 y 3 . Varios momentos en los que Teddy recuerda de manera distorsionada la realidad, mostrando a su mujer y su piso en llamas haciendo una referencia inconsciente a su piromanía.

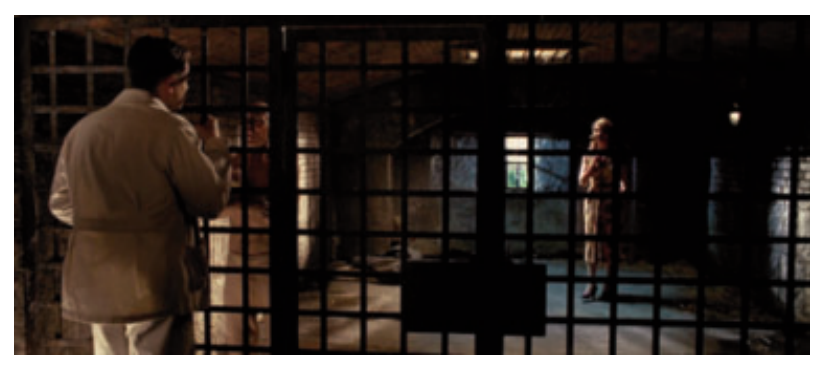

Foto 4. Teddy padeciendo una alucinación de su mujer en una de las escenas.

mentales. Entre ellos se encuentra el trastorno por estrés postraumático, donde son característicos los flashbacks y recuerdos que atormentan e incluso llegan a afectar físicamente al protagonista, con fuertes dolores de cabeza (Fotos 5 y 6 ).

Sin embargo, la principal patología aparente en Teddy es la esquizofrenia, que se presenta en forma de alucinaciones y delirios, principalmente personificados en la figura de su mujer.

Dentro de los tipos de ideas delirantes que presenta se deben destacar especialmente las de carácter
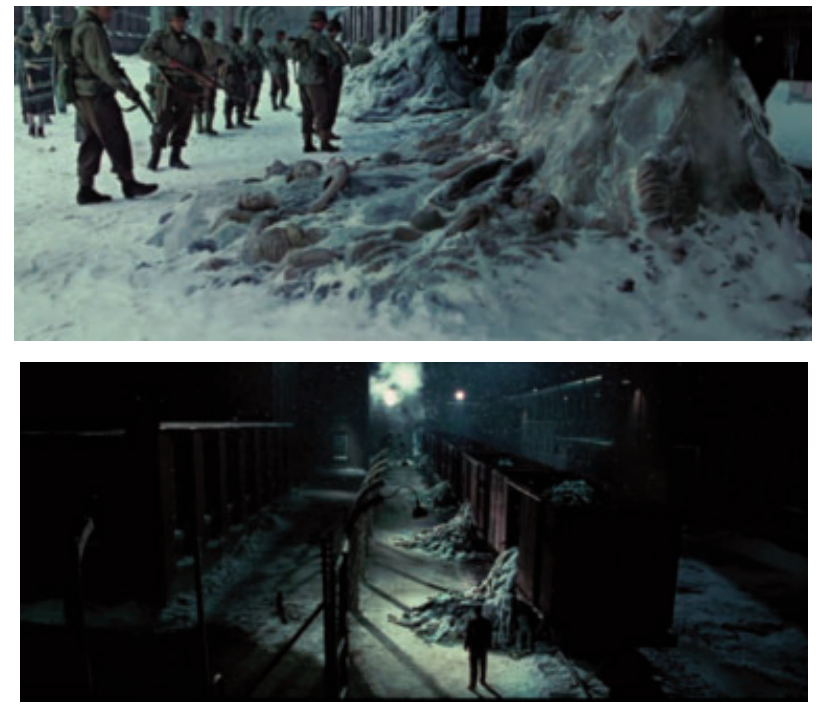

Fotos 5 y 6. Eescenas en las que Teddy recuerda el campo de concentración que su escuadrón.

persecutorio, manifestadas al sentirse perseguido y manipulado por algunas de las personas de su entorno; y las autorreferenciales, manifestadas a lo largo de la película al verse como un detective con un caso que debe resolver.

A medida que avanza la película, Teddy sufre distinta sintomatología física, como el aumento de la sudoración, nerviosismo, inquietud y temblores que van en aumento a medida que se desarrolla la trama. Esto se debe principalmente al síndrome de abstinencia del tratamiento con Clorpromazina, medicación que deja de tomar al pensar que todo se trata de una gran conspiración contra él (Foto 7).

Tras averiguar que todo se trata de un complejo psicodrama, como tratamiento alternativo a los fallidos y clásicos psicofármacos, el espectador es consciente de que Teddy es realmente un enfermo mental interno del psiquiátrico, y que todo el caso que está investigando no es más que una historia inventada por él mismo con el

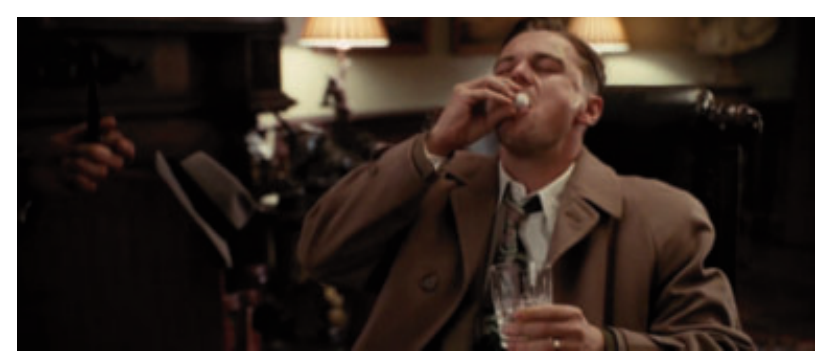

Foto 7. Teddy tomando la medicación que le ofrece el Dr. Cawley. 
principal objetivo de ocultar y olvidar los trágicos sucesos que rodeaban a la muerte de su familia.

Su mente trata de aliviar el dolor que le produce recordar que él mismo mató a su esposa Dolores Chanal, quien padecía un trastorno bipolar que llevó a ahogar a sus tres hijos en uno de sus brotes maníacos.

Teddy Daniels, o lo que es lo mismo; Andrew Laedis, inventa este último personaje, atribuyéndole su verdadero nombre y características negativas para eludir toda la responsabilidad y culpabilidad del crimen cometido, cargándolo en esta persona. Es por esto que se imagina a Andrew como un ser despreciable, macabro, con rasgos faciales deformados y con actitudes pirómanas y alcohólicas. Puesto que son todas estas características, aquellas de las que él mismo quiere desprenderse y reflejar en un personaje ficticio. Liberándose así de su culpabilidad y dolor y dándole un sentido a su vida; encontrar al "asesino de su familia" (Fotos 8, 9 y 10).

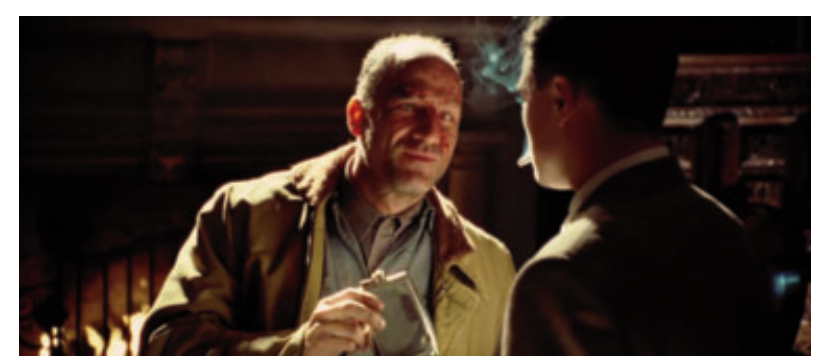

Foto 8. Andrew ofreciéndole alcohol a Teddy, haciendo referencia a su alcoholismo.
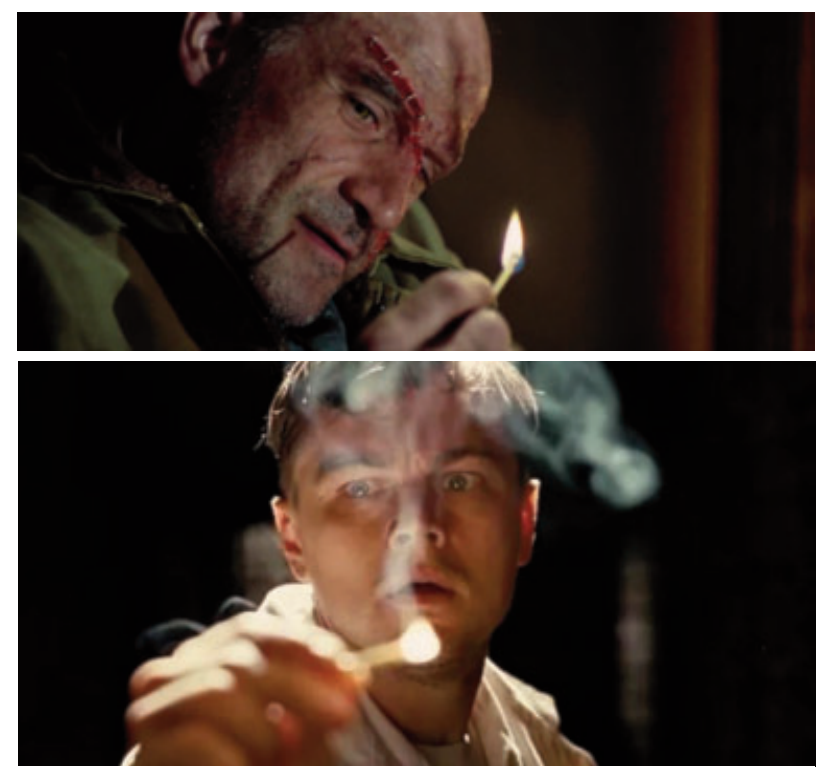

Fotos 9 y 10. Uso de la cerilla a modo de metáfora de piromanía y como paralelismo entre los dos personajes.
Es, por tanto, su propia mente la que crea la solución a su dolor, elaborando una historia ficticia donde nunca mató a su mujer. Por otra parte, es también la causante de su enfermedad; puesto que el estrés post traumático sufrido tras la Segunda Guerra Mundial, unida al alcoholismo que padecía tras esta y, finalmente, al trauma que supuso ver su familia destrozada, son las principales razones desencadenantes de su esquizofrenia (Fotos 11 y 12$)$.
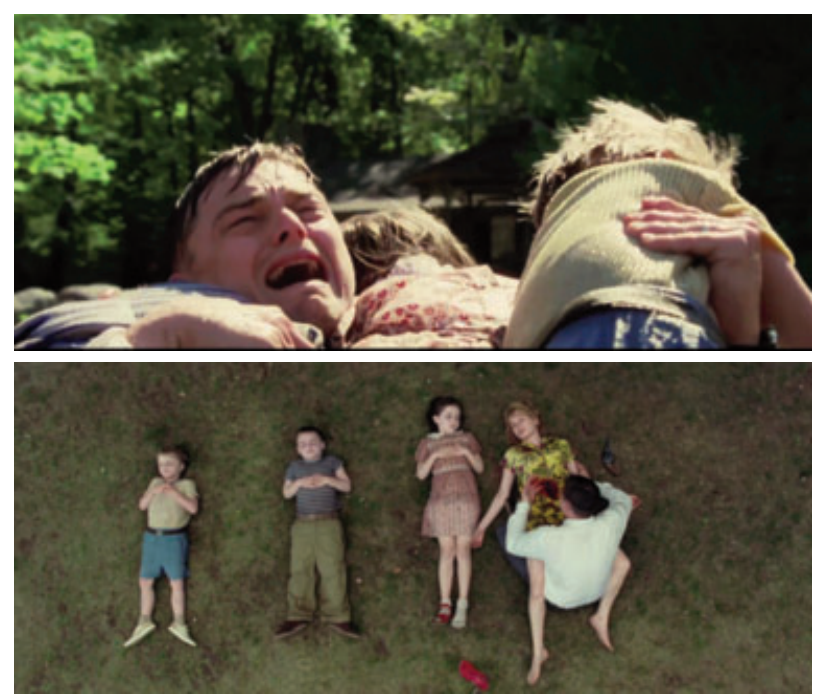

Fotos 11 y 12. Teddy encuentra a sus hijos muertos y acaba asesinando a su mujer.

Uno de los sucesos traumáticos que desencadenan la enfermedad de Teddy, puede equipararse a la patología mental clásica registrada en los veteranos de la Segunda Guerra Mundial, donde sus vivencias, especialmente de origen bélico, desembocaban en una serie de síntomas, principalmente: ansiedad, neurosis depresiva, melancolía y esquizofrenia. Al igual que en el caso de Teddy, estos trastornos se asociaban a la necesidad de estos excombatientes por aliviar el dolor que les suponía recordar todos aquellos sucesos tan traumáticos ${ }^{11}$.

Además, uno de los tratamientos más utilizados en estos veteranos fue el de la lobotomía11. Operación que tenía como propósito la eliminación de la patología mental y el comportamiento violento, dato que coincide con el caso de Teddy, puesto que sus alucinaciones le convierten en un sujeto peligroso para él mismo y para el resto de personas de la isla.

Acercándonos al final de la historia se desvela que su compañero del caso, Chuck Aule, es en realidad su psiquiatra, el doctor Sheehan. Este trata de evitar que se le 
realice una lobotomía a Teddy, para ello deja que sus delirios y alucinaciones fluyan de manera que llegue a comprender por sí mismo que no son verdaderas y pueda volver a la realidad. Para ello permite que el paciente viva su propia historia, a través del psicodrama antes mencionado, hasta que al final le desvelan, con pruebas lo suficientemente sólidas, que todo era una invención creada por su mente.

Esta terapia que se lleva a cabo con Teddy durante la película, es equiparable al tratamiento que más eficacia ha demostrado para reducir las complicaciones del estrés post traumático. Se trata de programas cognitivocomportamentales a través del proceso de inmersión en el relato traumático utilizando la Realidad Virtual. Algo que en la época que está ambientada la película es impensable, pero que a día de hoy permite guiar al psicoterapeuta la exposición y eliminar las limitaciones que el psicodrama en este caso puede presentar; como la evitación cognitiva de ciertos hechos que lleva a cabo Teddy o su propia capacidad de imaginación ${ }^{12}$.

Al final de la película cabe destacar la siguiente frase de Teddy: "Este lugar hace que me pregunte qué sería peor: vivir como un monstruo o morir como un hombre bueno". Lo que lleva a pensar que, quizás, el psicodrama había sido efectivo como terapia y Teddy realmente se había curado. Aunque es posible que este prefiera seguir fingiendo su patología mental al referirse al doctor Sheehan de nuevo como Chuck, su compañero de investigación ficticio, y al decirle que deben huir de la isla; solamente para que le practiquen la lobotomía y así no vivir con la culpa de haber asesinado a su mujer (Fotos 13 y 14).

\section{Conclusiones}

La esquizofrenia del protagonista se define como la respuesta de su mente a consecuencia de un conjunto de sucesos traumáticos; haciéndose necesaria para no sentirse un monstruo, algo de lo que trata de desprenderse mediante una clara disociación de personalidad.

Esta necesidad por olvidar se puede apreciar al final de la película, cuando el protagonista, tras ser consciente de su enfermedad y reconocer el asesinato que llevó a cabo, prefiere volver a fingir su enfermedad para ser lobotomizado y tener así la solución al dolor que la esquizofrenia le concedía durante el transcurso de su delirio.

Teniendo en cuenta que el estrés postraumático actúa como un factor de riesgo psicosocial para el desarrollo de esquizofrenia, como bien se demuestra en la
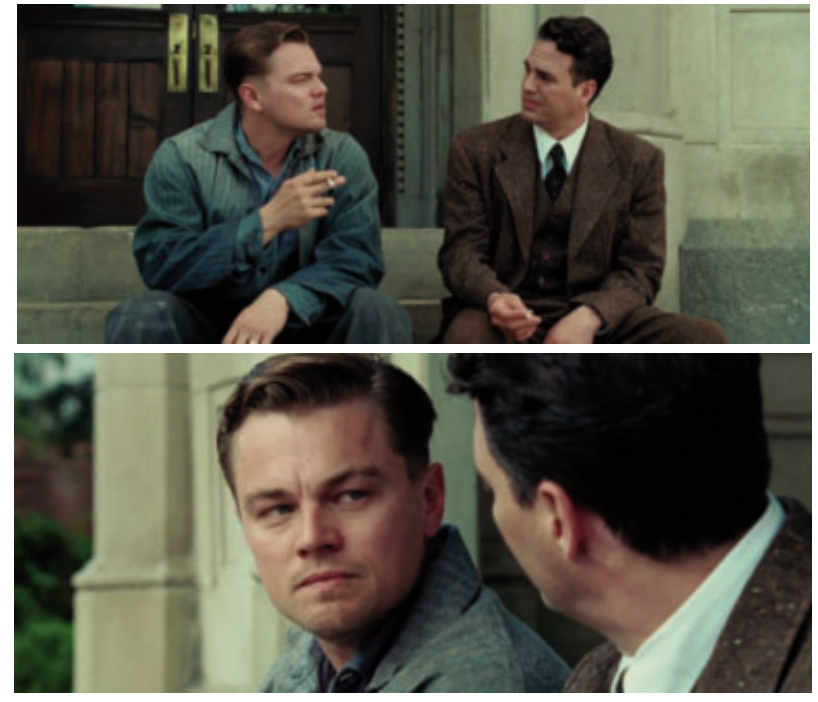

Fotos 13 y 14. Momento en el que Teddy dice la frase: "Este lugar hace que me pregunte qué sería peor: vivir como un monstruo o morir como un hombre bueno".

película y en los estudios llevados a cabo con excombatientes, es de vital importancia desarrollar más estudios alrededor de este factor etiológico; puesto que cuanto más se conozca sobre él, mayor capacidad de abordaje profesional se tendrá sobre la enfermedad. Ya que es el psicosocial el único de los factores de riesgo sobre el que podemos trabajar incidiendo en la prevención, tratamiento y recuperación.

Finalmente, se hace patente la necesidad de un abordaje psicológico de aquellas personas que han sufrido sucesos traumáticos, especialmente aquellos relacionados con sucesos bélicos y violentos, por el gran impacto emocional que suponen.

\section{Recomendaciones}

Como tratamiento se sugieren terapias individualizadas y grupales, con el principal objetivo de que expresen sus sentimientos y que, a su vez, sean capaces de asumir sus actos y perdonarse a sí mismos. En este tipo de casos los programas cognitivo-comportamentales han demostrado su eficacia a través del proceso de inmersión en el relato traumático ${ }^{12}$.

Por ello, a día de hoy se propone la Realidad Virtual como una buena alternativa para el procesamiento del acontecimiento traumático, ya que la simple imaginación presenta algunas limitaciones como la evitación cognitiva o la capacidad de imaginación.

De esta forma se lograría una exposición guiada y gradual al problema, pudiendo evitar que parte de los 
hechos traumáticos vividos actuasen como factores desencadenantes de otras enfermedades mentales, como la esquizofrenia.

\section{Referencias}

1. Ruiz M, Montes J, Correas J, Álvarez C, Mauriño J, de Dios C. Opiniones y creencias sobre las enfermedades mentales graves (esquizofrenia y trastorno bipolar). Rev Psiquiatr Salud Ment. 2012; 5(2):98-106.

2. Pérez-Álvarez M. Esquizofrenia y cultura moderna: razones de la locura Psicothema 2012;24(1):1-9.

3. Miret S, Fatjó-Vilas M, Peralta V, Fañanás L. Síntomas básicos en la esquizofrenia, su estudio clínico y relevancia en investigación. Rev Psiquiatr Salud Ment. 2012; 9(2):111-22.

4. Esquizofrenia [Internet]. Organización Mundial de la Salud. 9 de abril de 2018. 5. Estrategia en Salud Mental del Sistema Nacional de Salud. Madrid: Ministerio de Sanidad de España; 2007.

6. Iruin A, Aldama A, Ramírez M, López-Picado A, Latorre A, Etxebeste M, et al. Mapa geográfico de salud mental: variación geográfica en psicosis y asociación con factores ambientales. Vitoria-Gasteiz: Servicio Central de Publicaciones del Gobierno Vasco; 2014.

7. González Parra S, Molina J, Huertas D, Blasco-Fontecilla H, Mora-Mínguez F, Pascual-Pinazo F. Violencia asociada a los trastornos esquizofrénicos. Arch Psiquiatr. 2008;71(2-4): 109-27.

8. Grupo de Trabajo de la Guía de Práctica Clínica sobre la esquizofrenia y el trastorno psicótico incipiente. Fòrum de Salut Mental, coordinación. Guía de Práctica Clínica sobre la esquizofrenia y el trastorno psicótico incipiente. Versión resumida [Internet]. Madrid: Plan de Calidad para el Sistema Nacional de Salud del Ministerio de Sanidad y Consumo. Agència d'Avaluació de Tecnologia i Recerca Mèdiques; 2009.

9. Muñoz M, Pérez Santos E, Crespo M, Guillén A. Estigma y enfermedad mental: análisis del rechazo social que sufren las personas con enfermedad mental. Madrid: Editorial Complutense S.A; 2009.

10. Gejman Pablo V., Sanders Alan R. La etiología de la esquizofrenia. Medicina (Buenos Aires). 2012; 72(3): 227-34.

11. Alzate Palacio V. Un descanso a la "locura": La emergencia del Trastorno de Estrés Postraumático en los veteranos de la Segunda Guerra Mundial en EE.UU. y la lobotomía como solución desesperada. Tempus [Internet]. 2018; (1):62-78. 12. Botella C, García A, Baños RM, Quero S. Realidad Virtual y Tratamientos Psicológicos. C Med Psicosom. 2007; (82):17-31.
Josu Segura Abril. Estudiante de Tercer curso de Enfermería en la Universidad del País Vasco.

Iñigo Rolo Barinaga. Graduado en Ciencias de la Comunicación; especialidad: Publicidad y Relaciones Públicas en la Universidad del País Vasco. Estudiante de Tercer curso de Enfermería en la Universidad del País Vasco.

Oscar Parrado López. Estudiante de Tercer curso de Enfermería en la Universidad del País Vasco. 